EPJ Web of Conferences 66, 02072 (2014)

DOI: $10.1051 /$ epjconf/ 20146602072

(C) Owned by the authors, published by EDP Sciences, 2014

\title{
First observation of an isomeric state in proton drip-line nucleus ${ }^{26} \mathbf{P}$
}

D. Nishimura ${ }^{1, a}$, M. Fukuda ${ }^{2}$, T. Sakai ${ }^{3}$, M. Tanaka ${ }^{2}$, K.Abe $^{3}$, J. Chiba $^{1}$, S. Fukuda ${ }^{4}$, H. Furuki ${ }^{5}$, A. Homma ${ }^{3}$, H. Hotaka ${ }^{1}$, N. Ichihashi ${ }^{5}$, N. Inaba ${ }^{5}$, K. Imamoto ${ }^{2}$, T. Izumikawa ${ }^{6}$, Y. Kamisho ${ }^{2}$, K. $\mathrm{Kanbe}^{2}$, N. Kikukawa ${ }^{3}$, A. Kitagawa ${ }^{4}$, J. Kouno ${ }^{5}$, M. Nagashima ${ }^{3}$, Y. Nakamura ${ }^{3}$, I. Nishizuka ${ }^{5}$, K. Matsuta ${ }^{2}$, M. Mihara ${ }^{2}$, S. Miyazawa ${ }^{5}$, Y. Morita ${ }^{2}$, J. Ono ${ }^{2}$, T. Ohtsubo ${ }^{3}$, K. Sato ${ }^{5}$, S. Sato ${ }^{4}$, D. Sera ${ }^{3}$, S. Suzuki ${ }^{3,4}$, S. Suzuki ${ }^{5}$, T. Suzuki ${ }^{5}$, M. Takechi ${ }^{7}$, K. Tashiro ${ }^{3}$, M. Wakabayashi ${ }^{2}$, D. Watanabe $^{1}$, M. Yaguchi ${ }^{2}$, T. Yamaguchi ${ }^{5}$, S. Yamaki ${ }^{5}$, S. Yasumoto ${ }^{5}$, K. Yoshinaga ${ }^{1}$, and Y. Zhu ${ }^{1}$

${ }^{1}$ Department of Physics, Tokyo University of Science, Noda 278-8510, Japan

${ }^{2}$ Department of Physics, Osaka University, Toyonaka 560-0043, Japan

${ }^{3}$ Department of Physics, Niigata University, Niigata 950-2102, Japan

${ }^{4}$ National Institute of Radiological Sciences, Chiba 263-8555, Japan

${ }^{5}$ Department of Physics, Saitama University, Saitama 338-3570, Japan

${ }^{6} \mathrm{RI}$ center, Niigata University, Niigata 951-8510, Japan

${ }^{7}$ Gesellschaft für Schwerionenforschung GSI, 64291 Darmstadt, Germany

\begin{abstract}
An isomeric state in the proton drip-line nucleus ${ }^{26} \mathrm{P}$ has been observed by the $\gamma$-ray spectroscopy. The $\gamma$-ray energy and the half-life are $164.4 \pm 0.1 \mathrm{keV}$ and $120 \pm 9$ $\mathrm{ns}$, respectively. For the isomeric transition of the mirror nucleus ${ }^{26} \mathrm{Na}$, the $\gamma$-ray energy of $82.40 \pm 0.04 \mathrm{keV}$ and the half-life of $4.35 \pm 0.16 \mu$ s are also revised. Comparing the experimental reduced transition probabilities of ${ }^{26} \mathrm{Na}$ and ${ }^{26} \mathrm{P}$ with theoretical ones calculated by the shell model with the USDA interaction, the spin-parity $\left(J^{\pi}\right)$ of the isomeric state in ${ }^{26} \mathrm{P}$ is most likely to be $1^{+}$.
\end{abstract}

\section{Introduction}

The proton drip-line nucleus ${ }^{26} \mathrm{P}$ has attracted much interests since it is a candidate of proton halo nucleus due to the possible weakly-bound valence proton in $1 s_{1 / 2}$ state [1]. Furthermore, it is a key nucleus of the astrophysical problems such as the $r p$-process nucleosynthesis and X-ray bursts $[2,3]$. These studies are strongly related to the proton separation energy $\left(S_{p}\right)$ i.e. the mass and the $Q$ value of proton decay. The mass prediction from Audi et al. gives the atomic mass excess of ${ }^{26} \mathrm{P}$, $\Delta\left({ }^{26} \mathrm{P}\right)=10970 \pm 200 \mathrm{keV}$ (i.e. $S_{p}=144 \pm 200 \mathrm{keV}$ ) [4]. In the $\beta$-decay experiment [5], Thomas et al . deduced $S_{p}=0 \pm 90 \mathrm{keV}$ by using the Coulomb energy difference from ${ }^{26} \mathrm{Si}$ and the semi-empirical Isobaric multiplet mass equation (IMME) prediction [6]. On the other hand, the mirror nucleus ${ }^{26} \mathrm{Na}$ has a low-lying isomeric state at the excitation energy of $82.5 \pm 0.5 \mathrm{keV}$ [7]. The spin-parity $\left(J^{\pi}\right)$ and the half-life of the isomeric state are determined to be $1^{+}$and $9 \pm 2 \mu \mathrm{s}$, respectively. Assuming the mirror symmetry, there should be an isomeric state with $J^{\pi}=1^{+}$at the small excitation energies

ae-mail: dnishimura@rs.tus.ac.jp 


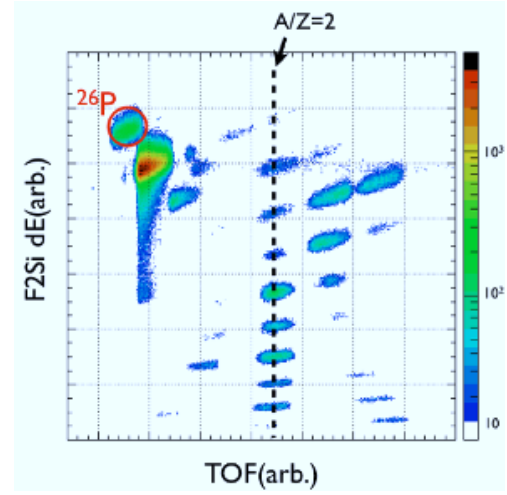

Figure 1. The particle identification plot via $B \rho$-TOF- $\Delta E$ method.

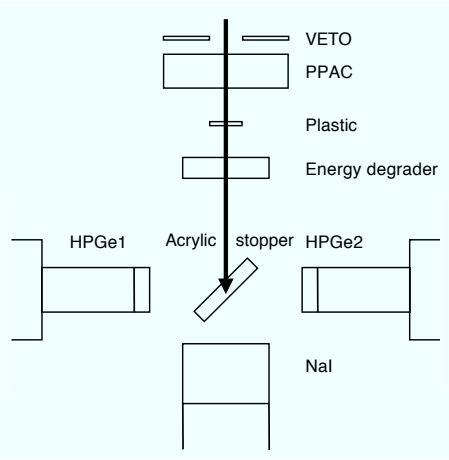

Figure 2. The $\gamma$-ray detection system.

in ${ }^{26} \mathrm{P}$. In this case, the isomeric state can also be the candidate of the proton halo nucleus and is strongly related to the astrophysical problems. However, if the excitation energy of the mirror state is much larger than $S_{p}$, it can decay by the fast direct proton emission. To investigate the existence of the isomeric state in ${ }^{26} \mathrm{P}$, the $\gamma$-ray spectroscopy has been carried out at NIRS (National Institute of Radiological Sciences)-HIMAC. Additionally, the excitation energy and the half-life of the isomeric state in ${ }^{26} \mathrm{Na}$ were precisely measured to discuss the isospin symmetry of transition probabilities.

\section{Experiment}

A primary beam of ${ }^{28} \mathrm{Si}$ with an energy of $300 \mathrm{MeV} / \mathrm{u}$ and with an intensity of $2 \times 10^{8}$ particles per spill (pps) was accelerated by the heavy-ion synchrotron at NIRS-HIMAC. Duration of each beam spill was $1 \mathrm{sec}$ and the repetition time was $3.3 \mathrm{sec}$. Secondary beams of neutron-deficient nuclei were produced by bombarding a $1.9-\mathrm{g} / \mathrm{cm}^{2}$-thick polyethylene target. They were separated and identified by passing through the secondary beam line (SB2) consisting of two dipole magnets and a $3.4-\mathrm{g} / \mathrm{cm}^{2}$ thick wedge-type $\mathrm{Al}$ degrader placed at the first focal plane (F1) between these magnets [8]. The particle identification (PID) was performed by $B \rho$-TOF- $\Delta E$ method. Figure 1 shows the PID plot with the time of flight (TOF) vs. the energy loss $(\Delta E)$. The TOF was measured by using two 0.5mm-thick plastic scintillators at F1 and the third focal plane (F3) which were $24 \mathrm{~m}$ apart along the beam line. The $\Delta E$ was measured by a $0.5-\mathrm{mm}$-thick $\mathrm{Si}$ detector placed at the second focal plane (F2) after the second magnet. The yield of ${ }^{26} \mathrm{P}$ was $30 \mathrm{pps}$ and the purity was $4 \%$, where the main contamination was ${ }^{25} \mathrm{Si}$. After passing through the SB2, the $100-\mathrm{MeV} / \mathrm{u}{ }^{26} \mathrm{P}$ beam was transported to the $\gamma$-ray detection system at the third focal plane. The schematic drawing of the system is shown in Fig.2. The beam penetrated through a plastic scintillator with a $20-\mathrm{mm} \phi$ hole (VETO), a parallel plate avalanche counter (PPAC) with an effective size of $100 \times 100 \mathrm{~mm}^{2}$, the plastic scintillator providing the stop signal for the TOF, and a $1.3-\mathrm{g} / \mathrm{cm}^{2}$-thick carbon energy degrader. Finally, the beam was implanted in the center of a $0.6-\mathrm{g} / \mathrm{cm}^{2}$-thick acrylic stopper. The acrylic stopper was tilted by 45 degrees with respect to the beam axis. The $\gamma$ rays emitted by the implanted nuclei were measured by two high-purity germanium (HPGe) detectors with 0.5 -mm-thick Be windows. The HPGe detectors 


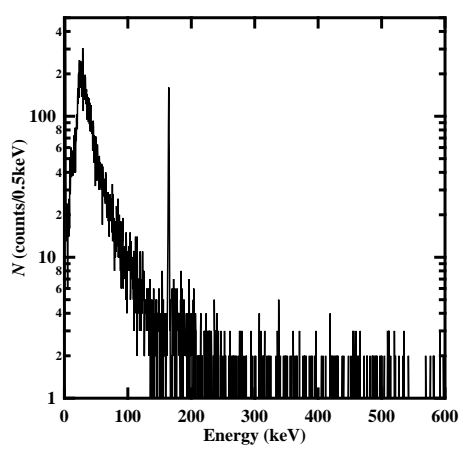

Figure 3. The $\gamma$-ray energy spectrum obtained in coincidence with the incoming signal of ${ }^{26} \mathrm{P}$ with a time gate of $2 \mu \mathrm{s}$.

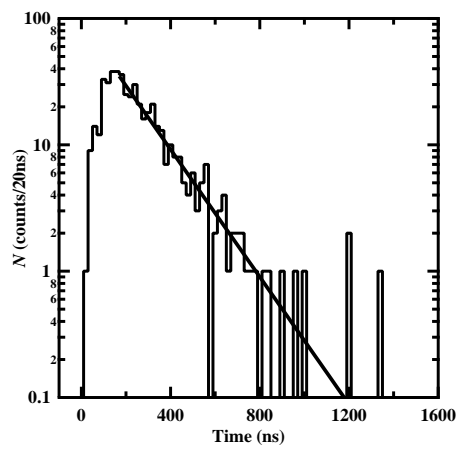

Figure 4. The time spectrum gated on the $164-\mathrm{keV}$ peak. The solid line represents the fitting result.

were set on both sides of the stopper and were placed perpendicular to the beam axis. The distances between both of the HPGe detectors and the stopper were $50 \mathrm{~mm}$. Figure 3 shows the $\gamma$-ray energy spectrum obtained in coincidence with the incoming signal of ${ }^{26} \mathrm{P}$ with a time gate of $2 \mu \mathrm{s}$. A peak at $164.4 \pm 0.1 \mathrm{keV}$ was clearly observed. Any other peaks associated with ${ }^{26} \mathrm{P}$ were not observed in our experiment. The $164-\mathrm{keV}$ peak disappeared in anti-coincidence with the signal of ${ }^{26} \mathrm{P}$. The time spectrum gated on the 164-keV peak is shown in Fig.4. The half-life of $120 \pm 9$ ns was determined by fitting the decay curve using the maximum likelihood method. The isomeric ratio after stopping in the acrylic stopper was determined to be $32 \pm 3 \%$, where the total absolute detection efficiencies for two HPGe detectors at $164 \mathrm{keV}$ were deduced to be $1.6 \%$ by using a checking source of ${ }^{133} \mathrm{Ba}$. Taking into account the inflight decay, the isomeric ratio at the production target was deduced to be $97_{-10}^{+3} \%$. This fact means that the branching ratio of the direct proton decay is at most $3_{-3}^{+10} \%$, even if the isomeric ratio at the production is $100 \%$.

As is the case for ${ }^{26} \mathrm{Na}$, the isomeric $\gamma$ rays were observed in the similar set up. A peak at $82.40 \pm 0.04$ $\mathrm{keV}$ was observed. The half-life of $4.35 \pm 0.16 \mu$ s was determined by fitting the decay curve. The present result of the half-life is not so inconsistent with the previous result of $9 \pm 2 \mu \mathrm{s}$, because the error of the previous value is very large.

\section{Discussion}

The observed $164-\mathrm{keV} \gamma$ rays for ${ }^{26} \mathrm{P}$ are not following the direct proton decay, because there are no excited states emitting the $\gamma$ rays with the same energy in ${ }^{25} \mathrm{Si}$. Therefore, it is concluded that the $164-\mathrm{keV} \gamma$ rays are originated from an isomeric state in ${ }^{26} \mathrm{P}$. Since other statistically significant peaks were not observed in this experiment, the $\gamma$ rays are possible to be emitted from the first excited state with an excitation energy of $164.4 \mathrm{keV}$ to the g.s. in ${ }^{26} \mathrm{P}$. It is also concluded that the isomeric state is below or not much higher than $S_{p}$ (i.e. proton decay threshold), because the branching ratio of the direct proton decay is nearly equal to zero. This is consistent with the results of $S_{p}=144 \pm 200$ $\mathrm{keV}$ by Audi et al. and $S_{p}=0 \pm 90 \mathrm{keV}$ by Thomas et al. The reduced transition probabilities for the isomeric state in ${ }^{26} \mathrm{P}$ can be evaluated in Weisskopf unit (W. u.) by using the observed excitation 
Table 1. Transition strengths for different multipolarity assumptions for $164-\mathrm{keV} \gamma$ decay in ${ }^{26} \mathrm{P}$.

\begin{tabular}{llllll}
\hline & $L=1$ & $L=2$ & $L=3$ & $L=4$ & $L=5$ \\
\hline Electric (W.u.) & $1.44 \times 10^{-6}$ & $8.58 \times 10^{0}$ & $7.75 \times 10^{7}$ & $1.04 \times 10^{15}$ & $1.95 \times 10^{22}$ \\
Magnetic (W.u.) & $4.13 \times 10^{-5}$ & $1.89 \times 10^{4}$ & $1.32 \times 10^{13}$ & $1.36 \times 10^{22}$ & $1.96 \times 10^{31}$ \\
\hline
\end{tabular}

Table 2. The experimental and theoretical $B(\mathrm{E} 2)$ values of ${ }^{26} \mathrm{P}$ and ${ }^{26} \mathrm{Na}$ in units of $e^{2} \mathrm{fm}^{4}$.

\begin{tabular}{llll}
\hline Nuclide & This work & Ref. [7] & Shell model calc. \\
\hline${ }^{26} \mathrm{P}$ & $39 \pm 3$ & - & 37.0 \\
${ }^{26} \mathrm{Na}$ & $30.0 \pm 0.7$ & $16_{-3}^{+5}$ & 41.9 \\
\hline
\end{tabular}

energy and the half-life. These values are summarized in Table 1, where the branching ratio of the proton decay and electron conversion are tentatively assumed to be zero. Since the reduced transition probability in units of $\mathrm{W}$. u. should not be extremely large, the multipolarities of E1, E2, and M1 are possible. Excluding the E1 and M1 transitions that show somewhat too much hinderance, the most probable transition type is E2. Assuming the transition type of pure E2 to the g.s. with $J^{\pi}=3^{+}$since an admixture of M1 would drastically increase the transition rate, it is expected that the isomeric state in ${ }^{26} \mathrm{P}$ has $J^{\pi}=1^{+}$or $J^{\pi}=5^{+}$. The shell model calculation using the USDA interaction [10] suggests that there are the first $1^{+}$state at $80 \mathrm{keV}$ and the first $5^{+}$state at $2240 \mathrm{keV}$ in ${ }^{26} \mathrm{P}$. Since the excitation energy of the isomeric state is similar to that of $1^{+}$state in the shell model calculation, it is probable that the isomeric state in ${ }^{26} \mathrm{P}$ has $J^{\pi}=1^{+}$.

Experimental and theoretical E2 transition probabilities $B(\mathrm{E} 2)$ for ${ }^{26} \mathrm{Na}$ and ${ }^{26} \mathrm{P}$ are summarized in Table 2. In the deduction of experimental values, $10 \%(1.6 \%)$ for ${ }^{26} \mathrm{Na}\left({ }^{26} \mathrm{P}\right)$ of the internal conversion coefficient were taken into account by using Kibédi's calculation [9]. In the shell model calculation, the effective charges for proton and neutron, $e_{p}=1.3 e$ and $e_{n}=0.5 e$, are used. The theoretical $B(\mathrm{E} 2)$ values well reproduces the present experimental $B(\mathrm{E} 2)$ values.

\section{Acknowledgments}

The present work was performed at NIRS-HIMAC under the research project with heavy ions. The authors are grateful to the staffs of HIMAC.

\section{References}

[1] Y.-J Liang et al., Chin. Phys. Lett. 26, 032102 (2009).

[2] K. Thielemann et al., astro-ph, 0101476 (2001).

[3] A. Parikh et al., Phys. Rev. C 79, 045802 (2009).

[4] G.Audi, A. H.Wapstra and C.Thibault, Nucl. Phys. A729, 337 ( 2003).

[5] J.-C. Thomas et al., Eur. Phys. J. A 21, 419 (2004).

[6] M. S. Antony et al., At. Data and Nucl. Data Tables, 34, 279 (1986).

[7] J. P. Dufor et al., AIP Conf. Proc. 164, 344 (1987).

[8] M. Kanazawa et al., Nucl. Phys. A 746, 393c (2004).

[9] Kibédi et al., Nucl. Instr. and Meth. A 589, 202 (2008).

[10] B. A. Brown and W. A. Richter, Phys. Rev. C 74, 034315 (2006). 